\title{
Asthma and smoking-induced airway disease without spirometric COPD
}

\author{
Neil C. Thomson \\ Affiliation: Institute of Infection, Immunity and Inflammation, University of Glasgow, Glasgow, UK. \\ Correspondence: Neil C. Thomson, Institute of Infection, Immunity and Inflammation, University of Glasgow, \\ Glasgow, G12 OYN, UK. E-mail: neil.thomsondaglasgow.ac.uk
}

@ERSpublications

Cigarette smokers without COPD develop chronic respiratory symptoms due to asthma and smokinginduced airway disease http://ow.ly/rTaS308SLll

Cite this article as: Thomson NC. Asthma and smoking-induced airway disease without spirometric COPD. Eur Respir J 2017; 49: 1602061 [https://doi.org/10.1183/13993003.02061-2016].

ABSTRACT Due to the high prevalence rates of cigarette smoking and asthma, current and ex-smokers frequently develop chronic airway disease without spirometric evidence of chronic obstructive pulmonary disease (COPD), either alone or associated with asthma. This review considers the classification, clinical outcomes, inflammatory and imaging variables, phenotypes, and management of current and ex-smokers with airway disease without COPD, focusing on overlaps in those with and without asthma. These individuals have more respiratory symptoms, worse quality of life, increased exacerbation rates, reduced lung function and more comorbidities than never-smokers with asthma or healthy never-smokers. As well as clinical features, airway inflammatory and structural changes in smoking-induced airway disease without COPD overlap with those found in smokers with asthma. Cigarette smoking is associated with worse clinical outcomes in some phenotypes of asthma. Management involves public health measures to control exposure to tobacco smoke, personal advice on smoking cessation and the use of appropriate targeted therapies, although evidence is limited on their effectiveness. Understanding the mechanisms, natural history and management of current and ex-smokers with asthma and smoking-induced airway disease without COPD is a priority for future research. 


\section{Introduction}

Asthma-chronic obstructive pulmonary disease (COPD) overlap syndrome (ACOS) is a term recently introduced to describe people with features of both asthma and COPD [1,2]. The definition is imprecise [3], since it is based on assessing the probability that clinical characteristics of both asthma and COPD are present in a patient with chronic airflow obstruction, defined as a forced expiratory volume in $1 \mathrm{~s}\left(\mathrm{FEV}_{1}\right)$ / forced vital capacity (FVC) ratio $<0.7$. The overlap syndrome is associated with worse clinical outcomes compared with COPD alone, including more severe symptoms, worse quality of life, more frequent exacerbations, greater decline in lung function, increased mortality and higher healthcare costs $[1,2,4,5]$. Estimates of the prevalence of ACOS range from $15 \%$ to $\sim 50 \%$ of patients with chronic airflow obstruction. ACOS is an umbrella term that describes a collection of phenotypes and endotypes of chronic airway disease, including subgroups with type 2 inflammation and eosinophilia [6]. Cigarette smoking is an important risk factor associated with the development of COPD and ACOS [7, 8]. Smoking-induced airway disease in people who do not have spirometric evidence of COPD is also recognised, either alone or in association with asthma. These subgroups of chronic airway disease with normal lung function are likely to occur commonly, due to the high prevalence rates for cigarette smoking and asthma in most populations. Worldwide, over 1 billion people are estimated to use tobacco products, mainly through smoking cigarettes, and over 300 million people have asthma. In many countries the proportion of cigarette smokers among people with asthma is similar to the general population [9], although in the USA, a higher percentage of people with asthma are smokers (21\%) than among those without asthma (16.8\%) [10]. Smoking-induced effects on the lungs, such as chronic bronchitis, emphysema, gas trapping and small airway disease, can occur in the absence of chronic airflow obstruction. Taken together, these findings suggest that chronic respiratory symptoms in current or ex-smokers without spirometric evidence of COPD could be due to asthma, smoking-induced airway disease or a combination of these conditions. This review considers the classification, clinical outcomes, inflammatory and imaging variables, phenotypes, and management of current and ex-smokers with airway disease without COPD, including those with and without asthma, and focuses on the overlaps between these subgroups.

\section{Epidemiological and clinical studies}

Epidemiological studies in the general population, observational studies of volunteers recruited to large cohorts used to investigate COPD, and studies of people with asthma in primary and secondary care provide information to help classify chronic airway disease in current and ex-smokers without spirometric COPD, including those who also have asthma, and to describe clinical, inflammatory and imaging variables in these individuals. Epidemiological studies of people randomly selected from the general population include the US Third National Health and Nutrition Examination Surveys (NHANES III) in 1988-1994 and 2007-2010 [11-13], the Canadian Cohort of Obstructive Lung Disease (CanCOLD) study in nine urban sites in Canada [14], and the New Zealand Wellington Respiratory Survey [15]. Information on current and ex-smokers without COPD is provided from subgroups of middle-aged and older volunteers recruited to the Genetic Epidemiology of COPD (COPDGene) study [16] and the Subpopulations and Intermediate Outcome Measures in COPD Study (SPIROMICS) [17, 18]. Both studies included individuals with a concomitant diagnosis of asthma. Data on current and ex-smokers with a diagnosis of asthma are

BOX 1 Key messages

- Due to the high prevalence of cigarette smoking and asthma, current and ex-smokers frequently develop chronic airway disease without spirometric evidence of chronic obstructive pulmonary disease (COPD), either alone or in association with asthma

- Adverse health-related outcomes occur due to smoking-induced airway diseases /chronic bronchitis, emphysema, small airway disease), asthma or a combination of these diseases

- Current and ex-smokers without COPD, either with or without asthma, have more respiratory symptoms, worse quality of life, increased exacerbation rates, reduced lung function and more comorbidities than never-smokers with asthma or healthy never-smokers

- As well as clinical features, airway inflammatory and structural changes in smoking-induced airway disease without COPD overlap with those found in smokers with asthma

- Cigarette smoking is associated with worse clinical outcomes in some phenotypes of asthma

- Management involves public health measures to control exposure to tobacco smoke, personal advice on smoking cessation and the use of appropriate targeted therapies, although evidence is limited on the effectiveness of therapeutic interventions

- Understanding the mechanisms, natural history and management of current and ex-smokers with airway disease without spirometric evidence of COPD, either alone or in association with asthma, is a priority for future research 
based on epidemiological studies in the general population, such as the Copenhagen General Population Study [19], and studies in primary care and secondary care [20-23]. Several important issues about methodology influence the interpretation of the evidence provided by these studies (table 1), such as differences in demographic factors between study populations, uncertainty about the diagnosis of the type of chronic airway disease, variations in exposure to tobacco smoke, differences in the methods used to assess clinical outcomes and generalisability of the findings.

TABLE 1 Methodological issues that influence the interpretation of studies of adults with asthma and smoking-induced airway disease without spirometric evidence of chronic obstructive pulmonary disease (COPD)

\section{Demographic information}

\section{Diagnosis of chronic airway disease}

\section{Smoking status}

\section{Assessment of clinical outcomes}

\section{Generalisability of findings}

Adult participants recruited to clinical studies of smokers with asthma are generally younger than those included in epidemiological studies in the general population and COPD cohorts were participants are middle-aged and older

Sex and race of participants may not be representative of the general population

Establishing an accurate diagnosis of asthma, smoking-induced airway disease without asthma, chronic bronchitis, emphysema or a combination of airway diseases can be difficult in symptomatic current smokers and ex-smokers without spirometric COPD

Asthma and other airway diseases may be underdiagnosed or misdiagnosed in observational studies that are based on history alone and without objective tests

The specificity of objective criteria used to diagnose asthma may not be sufficient to exclude people with symptomatic smoking-induced airway disease who do not have asthma

Observational studies in asthma can often report outcomes that combine data from smokers with and without chronic airflow obstruction (forced expiratory volume in $1 \mathrm{~s} /$ forced vital capacity $<0.7$ ), making it harder to assess the findings in those without spirometric COPD

Undiagnosed emphysema and air trapping can occur in cigarette smokers without spirometric COPD, and these conditions may be missed by spirometry and only become evident with additional tests of lung functions (transfer factor, whole-body plethysmography, impulse oscillatory, multiple-breath washout tests) and imaging techniques (low-dose computed tomography)

Studies may include people with other chronic respiratory diseases, such as bronchiectasis

Spirometric measurements can vary over time resulting in an individual being considered not to have COPD on recruitment to a study, when subsequent tests demonstrate airflow obstruction

The influence of factor(s) inducing airway disease other than tobacco smoke, such as occupational agents and environmental exposures, are not controlled in most studies

Symptoms due to upper airway disease can be misdiagnosed as being due to lower airway disease Combined data from current smokers and ex-smokers may obscure differences in outcomes in one or another smoking group

Findings in studies with an entry criteria of a smoking history of $\geqslant 10$ pack-years, such as COPDGene and SPIROMICS, may not be relevant to those with a less intense history of exposure to tobacco smoke [16-18]

Factors relating to smoking behaviour, such as number of puffs per cigarette, depth of inhalation and types of cigarette, may not be reflected by history of pack-years and current smoking status

Measures of current symptoms and quality of life in smokers with asthma, such as the ACT, ACQ and $A Q L Q$ scores, can overlap with those used to measure chronic respiratory symptoms and health status in smoking-induced airway disease with or without COPD, such as the CAT and SGRQ scores.

For example, the SGRQ score is associated with changes in AQLQ and by some treatments for asthma, such as mepolizumab, and concomitant asthma can confound CAT scores in current or ex-smokers [121]

The definition of chronic bronchitis varies between studies and in some, is based on components of the SGRQ score

The definition of severe exacerbation in asthma, which is based on the use of high-dose systemic corticosteroid for $\geqslant 3$ days, either alone or associated with an emergency department visit or hospital admission, overlaps with components of the definition of exacerbation-like respiratory symptoms used in studies of smoking-induced airway disease with or without COPD

In observation studies, the assessment of clinical outcomes can be influenced by recall bias

Age of onset of asthma influences the effects of cigarette smoking on lung function and possibly other variables

The selection process and criteria used to recruit participants to studies of airway disease in individuals without COPD may not be generalisable to the general population, although baseline demographic and clinical variables in the COPDGene study showed some similarities to those in the National Health and Nutrition Examination Survey study in the general population [16] 


\section{Classification of chronic airway diseases in smokers without COPD}

The ability to classify smoking-induced airway disease without spirometric COPD, with or without features of asthma, is hampered by the relatively small number of published studies, by the methodological factors as discussed in the previous section, and by a limited understanding of the phenotypes, endotypes and mechanism of airway disease in these subgroups. Epidemiological studies of middle-aged and older individuals without spirometric evidence of COPD, approximately half of whom are current or ex-smokers $[12-15,19]$, suggest that $14-20 \%$ have chronic airway disease, mainly due to asthma, chronic bronchitis or a combination of these diseases. A small proportion have emphysema alone, although this increases to one-third in heavy cigarette smokers [24]. In the COPDGene [16] and SPIROMICS [17] cohorts of symptomatic and asymptomatic middle-aged and older current and ex-smokers with normal spirometry, $11.4 \%$ and $18 \%$ had a history of asthma, $12.6 \%$ and $18 \%$ had chronic bronchitis, and $10.4 \%$ and $0 \%$ had computed tomography (CT) emphysema, respectively. Among the symptomatic current and ex-smoker subgroups, many had airway disease not associated with a history of asthma, although around one-third had asthma, one-third had chronic bronchitis and a small proportion had a combination of these conditions. Cluster analysis performed in two Korean cohorts of patients with asthma that included current smokers identified a "smoking asthma" cluster that was predominately male and had well-preserved FEV1 [25].

A classification of chronic airway disease is proposed for symptomatic current and ex-smokers without airflow obstruction (figure 1). Smoking-induced airway diseases include chronic bronchitis, emphysema, small airway disease or a combination of these diseases [26,27] and these conditions occur in people who also have a diagnosis of asthma. Some individuals may have undiagnosed asthma or other smoking-induced abnormality not detected by current techniques. Chronic respiratory symptoms in current and ex-smokers with asthma may be due to mechanisms induced by asthma, by smoking or by a combination of both pathways. Cigarette smoking is a risk factor for cardiovascular comorbidities, increased mortality and the development of asthma in adults [27, 28]. Risk factors implicated in causing COPD other than cigarette smoking [29], including exposure to biomass fuels, occupational agents and air pollution, as well as to impaired lung growth in childhood and poverty $[1,29]$, may contribute to airway damage in current and ex-smokers with normal spirometric lung function or with asthma. Lung disorders, such as interstitial lung disease, or nonrespiratory conditions, such as cardiac disease or high body mass index, can be alternative causes of symptoms in current and ex-smokers, and these disorders can occur in addition to chronic airway disease.

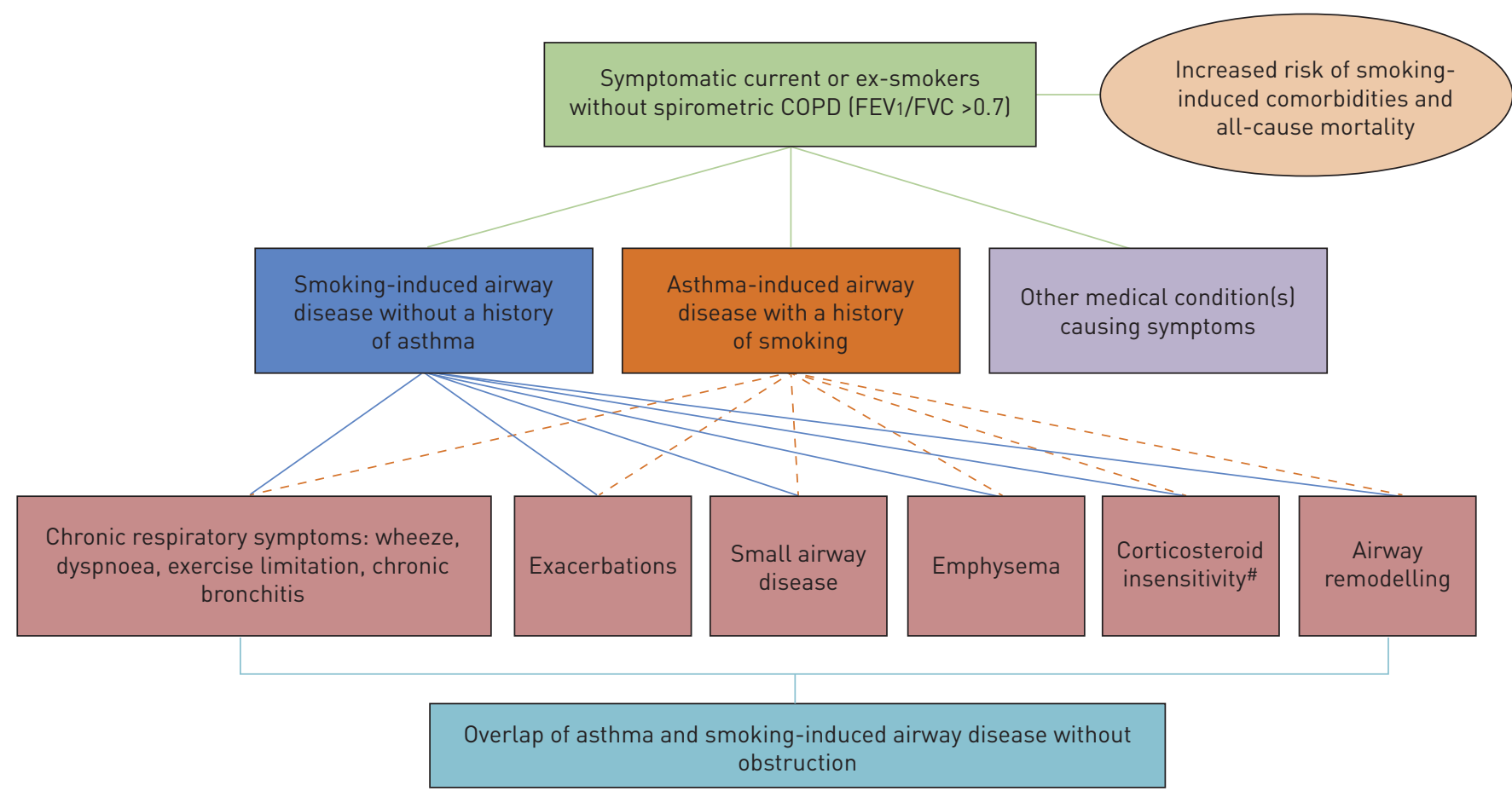

FIGURE 1 Classification of chronic airway diseases in symptomatic current and ex-smokers without spirometric airflow obstruction (forced expiratory volume in $1 \mathrm{~s} /$ forced vital capacity $\left.\left(F E V_{1} / F V C\right)>0.7\right)$ due to smoking-induced airway disease without a history of asthma, asthma-induced airway disease or an overlap of both diseases. ${ }^{\#}$ : it is not known whether current and ex-smokers with smoking-induced airway disease without evidence of chronic obstructive pulmonary disease (COPD) or a history of asthma have clinical evidence of corticosteroid insensitivity. 
In summary, classification of asthma and smoking-related diseases in current and ex-smokers without evidence of spirometric COPD will require to be revised in the light of future studies that provide a greater understanding of the underlying phenotypes and endotypes of chronic airway disease in these individuals.

\section{Clinical outcomes}

Similarities and differences in clinical outcomes in current and ex-smokers with chronic airway disease without spirometric COPD or a history of asthma are compared with current and ex-smokers with asthma (table 2).

\section{Chronic respiratory symptoms}

Chronic respiratory symptoms of wheezing and dyspnoea are experienced by between $15 \%$ and $30 \%$ of adults aged $\geqslant 40$ years in the general population, particularly in current and ex-smokers $[12,14,15]$. In the COPDGene and SPIROMICS cohorts, a history of wheezing, breathlessness or limitation of exercise was reported by approximately half the current and ex-smokers without COPD $[16,17,30]$. In the symptomatic subgroup of the SPIROMICS study, a high proportion reported wheezing (69\%) and had a diagnosis of asthma (27\%) [17]. Surveys of people with asthma, including those with severe disease, demonstrate that current smoking is an important risk factor for poor symptom control, including wheezing and breathlessness [19, 20, 31, 32], and these individuals often report limitations in exercise tolerance.

The prevalence rate for chronic bronchitis in the general population is $6.4 \%$ [33], with higher rates in smokers without airflow obstruction [34, 35], including adults aged $\geqslant 40$ years [12, 14, 15]. Chronic bronchitis was reported by $12.6 \%$ and $19 \%$ of current and ex-smokers with normal spirometry recruited to the COPDGene [16] and SPIROMICS cohorts [17], respectively, with a higher proportion reporting chronic bronchitis among symptomatic individuals (33\%) [17]. There is relatively limited information on the prevalence of chronic bronchitis in asthma [35-39]. The Copenhagen City Heart Study and the European Community Respiratory Health survey reported prevalence rates of $39 \%$ and $42 \%$, respectively, in nonsmokers with asthma $[35,37]$, with higher rates in smokers with asthma $[35,37]$, particularly those with severe disease [39]. Smoking-induced chronic bronchitis without COPD is a risk factor for respiratory exacerbations [14], and is associated with worse quality of life, reduced exercise tolerance and a higher pack-year history [40]. In smokers with asthma, chronic bronchitis is associated with worse current symptom control [39] and a greater decline in FEV1 [35].

\section{Quality of life}

Health-related quality of life is poorer in adults aged $\geqslant 40$ years without a diagnosis of asthma, emphysema or COPD who gave a history of exacerbations than those without exacerbations [14]. In the COPDGene

TABLE 2 Summary of the effects of smoking status on clinical outcomes in asthma and symptomatic smokers without spirometric chronic obstructive pulmonary disease (COPD) ${ }^{\#}$

Outcome
Asthma with a smoking history and without spirometric COPD: current smokers with asthma compared with never-smokers with asthma
Smoking-induced airway disease without spirometric COPD (no history of asthma): current and ex-smokers compared with healthy never-smokers

\begin{tabular}{|c|c|c|}
\hline \multicolumn{3}{|l|}{ Respiratory symptoms } \\
\hline Wheeze, breathlessness & Increased & Increased \\
\hline Exercise tolerance & Decreased & Decreased \\
\hline Chronic bronchitis & Increased & Increased \\
\hline Quality of life & Decreased or similar & Decreased \\
\hline Exacerbations & Increased or similar & Increased \\
\hline Healthcare utilisation & Increased & Increased \\
\hline \multicolumn{3}{|l|}{ Lung function } \\
\hline Baseline spirometry & Decreased & Decreased \\
\hline Small airway dysfunction & Increased & Increased \\
\hline Decline in FEV1 & Increased or similar & Similar \\
\hline \multicolumn{3}{|l|}{ Therapeutic response to ICSs } \\
\hline Short- to medium-term treatment & Decreased & Not known \\
\hline Long-term treatment & Decreased or similar & Not known \\
\hline Smoking-related comorbidities & Increased & Increased \\
\hline All-cause mortality & Increased & Increased \\
\hline
\end{tabular}

FEV1: forced expiratory volume in $1 \mathrm{~s}$; ICS: inhaled corticosteroid. * : refer to the main text for information on the published studies used to summarise the effects of smoking status on clinical outcomes in asthma and symptomatic smokers without spirometric COPD. 
cohort, current and ex-smokers without spirometric COPD had worse health status as assessed by the St George's Respiratory Questionnaire total score than healthy never-smokers [16]. In some surveys, smokers with asthma had worse levels of asthma-specific quality of life than never-smokers with asthma [41].

\section{Exacerbations and healthcare utilisation}

In the CanCOLD general population study, $\sim 4 \%$ of individuals without evidence of COPD or a history of asthma had an exacerbation in the last year [14], and these events were associated with female sex, presence of wheezing, the use of respiratory medications and self-perceived poor health. A 5-year follow-up of the COPDGene cohort reported that $4.1 \%$ of current and ex-smokers without COPD had one or more exacerbations per year and $\sim 1 \%$ had a severe exacerbation requiring hospital admission [42]. Risk factors for acute episode of respiratory disease in smokers without COPD are similar to those with COPD, although the rate of episodes is higher in the latter group [43]. In the SPIROMICS population, symptomatic current and ex-smokers more often had exacerbations $\left(0.27\right.$ events year $\left.{ }^{-1}\right)$ than asymptomatic current or ex-smokers $\left(0.08\right.$ events.year $\left.{ }^{-1}\right)$ or never-smokers $\left(0.03\right.$ events·year $\left.^{-1}\right)$ [17]. Current smoking is a risk factor for severe asthma exacerbations [27] including during pregnancy [44], and in severe disease [31], more hospitalisation for asthma [41], more visits to the emergency room for asthma [45] and increased numbers of life-threatening asthma attacks. In contrast, the Copenhagen General Population Study reported that the risk of asthma exacerbations was unrelated to smoking status [19].

\section{Lung function}

Spirometric lung function tests are reduced in cigarette smokers without a history of lung disease compared with never-smokers [1], in symptomatic current or ex-smokers without COPD compared with asymptomatic current or ex-smokers [17] and in smokers with asthma compared with never-smokers with asthma [19, 31, 46]. Small airway dysfunction occurs in current and ex-smokers without COPD, irrespective of the presence or absence of respiratory symptoms [18], and its presence is associated with a more rapid decline in lung function [47]. Tests of small airway function are also impaired in smokers with asthma [46]. In the COPDGene cohort, current and ex-smokers without spirometric COPD (FEV1/FVC greater than lower limit of normal) had abnormal forced expiratory volume in 3 and $6 \mathrm{~s}$ (FEV3/FEV6) ratios that identified individuals with mild COPD characterised by CT small airway disease, impaired exercise tolerance and reduced quality of life [48]. In a 5-year follow-up of the COPDGene cohort, the rate of FEV1 decline was similar in current and ex-smokers without COPD who reported breathlessness or exacerbations compared with those who did not [42]. Several longitudinal population-based studies report accelerated decline in lung function in adult smokers with asthma [35, 49, 50], although other studies report a similar decline in FEV1 in asthma irrespective of smoking status [37]. A greater proportion of smokers with adult-onset asthma develop persistent airflow obstruction and although current smokers with a history of childhood-onset asthma can develop COPD, this occurs in association with severe disease in childhood rather than cigarette smoking in adulthood $[51,52]$.

\section{Corticosteroid insensitivity}

Corticosteroid insensitivity occurs in chronic inflammatory diseases including severe asthma and COPD $[53,54]$. The effects of budesonide on epithelial barrier function in primary bronchial epithelial cells is impaired in asthma and COPD, and this effect is less evident in healthy smokers, suggesting that corticosteroid responsiveness in smokers without spirometric evidence of COPD might be better preserved than in those with COPD [55], although this hypothesis remains to be tested in clinical studies. Cigarette smokers with mild to moderate asthma are less sensitive to the short- and medium-term therapeutic effects of inhaled corticosteroids on symptoms and lung function compared with nonsmokers with asthma [21, 56, 57]. Smokers with chronic asthma are also less sensitive to short-term oral corticosteroid therapy [58] and to the cutaneous vasoconstrictor response to topical corticosteroids [59]. Data is conflicting on whether the long-term therapeutic effects of inhaled corticosteroids are impaired in smokers with asthma [60-64]. Taken together, these studies suggest that most smokers with asthma are less sensitive to short- and medium-term treatment with inhaled corticosteroids compared with nonsmokers with asthma, but that a small proportion of smokers can respond adequately to this treatment and that long-term treatment may have beneficial effects.

\section{Comorbidities and mortality}

Cigarette smoking is a risk factor for diseases that can impact on the health of smokers with asthma or smoking-induced airway disease without COPD, such as cardiovascular disease [65], anxiety and depression, and osteoporosis. In the US Multi-Ethnic Study of Atherosclerosis study, greater emphysema-like changes on cardiac CT were associated with increased all-cause mortality among people without airflow obstruction, particularly among smokers [66]. Comorbidities occur frequently in smokers with asthma, with higher rates of cardiovascular disease, pneumonia and lung cancer compared with never-smokers without asthma [19]. 
Patients with adult-onset asthma, at least one comorbidity and evidence of systemic inflammation, characterised by elevated levels of interleukin (IL)-6 and high-sensitivity C-reactive protein, had a higher pack-year history than patients without systemic inflammation or a comorbidity [67]. The NHANES I Follow-up Study reported an increased risk of respiratory mortality and all-cause mortality in asthma that may be explained by the confounding effect of cigarette smoking [68].

\section{Clinical outcomes in ex-smokers}

In the COPDGene cohort, ex-smokers had less chronic respiratory symptoms, fewer exacerbations and less airway wall area thickening plus greater emphysema and gas trapping than current smokers [16]. In the SPIROMICS cohort, the proportion of current smokers with chronic respiratory symptoms and exacerbations was similar to the ex-smokers [17]. In most studies, ex-smokers with asthma had levels of symptom control, chronic bronchitis, quality of life and healthcare use for asthma that were either similar or worse than never-smokers with asthma and that were generally better than current smokers with asthma [20,31, 37, 41, 69]. In one study of people with severe asthma, increased blood neutrophil counts in ex-smokers with a low pack-year history were associated with frequent exacerbations, whereas elevated blood eosinophils predicted exacerbations in never-smokers [70]. The Copenhagen General Population Study reported that the frequency of any respiratory symptoms was not associated with smoking status in individuals with asthma [19]. All-cause mortality and rates of cardiovascular disease, pneumonia and lung cancer in ex-smokers with asthma were similar to never-smokers and current smokers with asthma [19].

In summary, current and ex-smokers without COPD have more chronic respiratory symptoms, worse quality of life, increased exacerbation rates, reduced lung function and more comorbidities than never-smokers without COPD. There is considerable overlap in the range and frequency of adverse clinical outcomes between those with and without a diagnosis of asthma.

\section{Inflammatory and imaging variables}

Cigarette smoking and asthma induce distinct inflammatory and structural changes to the lungs, although less is known about whether these stimuli interact. Tobacco smoke induces pro-inflammatory responses in the lungs that are mediated by alveolar macrophages, neutrophils and respiratory epithelial cells, and it also impairs innate defence mechanisms that are produced by alveolar macrophages, epithelial cells, natural killer cells and dendritic cells [71]. The risk of developing smoking-related inflammation increases with greater smoking intensity in healthy subjects [72]. The bronchial mucosa of asymptomatic smokers without COPD has increased inflammatory cells, including neutrophils, mast cells and macrophages, increased cells staining positive for IL-1 $\beta$ and IL-8, reduced epithelial integrity, and increased thickness of the laminin layer in the subepithelium [73, 74]. In smokers with normal lung function, it is likely that epithelial inflammation and glandular hyperplasia contribute to symptoms of chronic bronchitis, impaired inflammatory responses contribute to increased risk of infection and altered immunity contributes to airway damage. Published data are not available on the airway histology in symptomatic and asymptomatic smokers without spirometric COPD recruited to the COPDGene and SPIROMICS cohorts. Current smokers without COPD, but with evidence of small airway dysfunction, have evidence of distal airway inflammation $[75,76]$. In the CanCOLD study of middle-aged or older current and ex-smokers with normal lung function (mean age 68 years), the prevalence of bronchial wall thickening, air trapping and emphysema on visual inspection of CT scans was 58\%, 35\% and 30\%, respectively, compared with never-smokers with no obstruction [24]. CT emphysema was estimated to be mild, since it was not associated with worse clinical outcomes [24]. In the COPDGene cohort, approximately one-third of current and ex-smokers without spirometric COPD had segmental CT airway wall thickening [16]. In the SPIROMICS population, symptomatic current and ex-smokers without spirometric evidence of COPD, irrespective of a history of asthma, had greater CT airway wall thickening compared with asymptomatic individuals [17]. Quantitative CT and visual scoring emphysema was reported in $10.4 \%$ and $24 \%$, respectively, of current and ex-smokers without spirometric COPD recruited to COPDGene [16].

In smokers with asthma, sputum airway inflammation is often reported to be noneosinophilic, either neutrophilic or paucigranulocytic [21, 22, 31], although a recent study found no association between smoking status and sputum inflammatory phenotypes in asthma [77]. Differences in the intensity of smoking may explain some of the discrepancies between studies. Exhaled nitric oxide concentration is reduced in current cigarette smokers with asthma [31,78], whereas concentrations are similar in ex- and never-smokers with severe asthma [31]. Bronchial biopsy studies in smokers with mild and moderate asthma report reduced eosinophil numbers, increased submucosal mast cell numbers [79] and increased $\mathrm{CD}^{+}$T-lymphocytes [80] compared with never-smokers with asthma. In addition, smokers with asthma have increased bronchial goblet cell numbers and more proliferation and greater thickness of the epithelium [79]. In the U-BIOPRED cohort of individuals with severe asthma, CD4 ${ }^{+}$lymphocyte numbers 
were reduced in current and ex-smokers compared with nonsmokers with severe asthma [81]. Airway smooth muscle mass, basement membrane thickness and submucosal gland area are similar [79, 82]. In keeping with these latter findings, CT measures of segmental airway wall thickness are similar in smokers and never-smokers with mild to severe asthma [46]. CT large airway luminal area is reduced in smokers with severe asthma [46], particularly in those who gave a history of chronic mucus hypersecretion [39], possibly due to mucus accumulation in the large airways and to structural changes to the airways. CT emphysema is an infrequent finding in smokers with asthma [46].

In summary, these observations suggest that tobacco-induced airway inflammatory and structural changes are present in smokers without spirometric COPD compared with healthy never-smokers. Airway inflammation in smokers with asthma differs from that of never-smokers with asthma; current smoking is associated with less eosinophilic airway inflammation, more mast cell numbers, increased bronchial epithelial glands and reduced segmental airway luminal area. Airway inflammatory and structural changes in smoking-induced airway disease without COPD overlap those found in smokers with asthma.

\section{Phenotypes}

Subtypes or phenotypes of asthma are recognised, based on clinical, physiological or immunological variables, and these are used to classify asthma and to predict response to therapy [83]. Phenotypes of smoking-related disease occur in current and ex-smokers without spirometric COPD or a history of asthma, such as chronic bronchitis, emphysema and small airway disease (figure 1). Current and former cigarette smoking in asthma is associated with worse outcomes in certain clinical phenotypes discussed earlier, such as chronic bronchitis, chronic persistent airflow obstruction and corticosteroid insensitivity. There is limited and, in some cases, conflicting information on the influence of current and former cigarette smoking on clinical and inflammatory phenotypes of asthma.

\section{Clinical phenotypes}

\section{Adult-onset asthma}

Greater pack-year history is a predictor of uncontrolled asthma, increased disease severity and greater decline in lung function in people with adult-onset asthma [84-88]. A higher proportion of people with severe adult-onset asthma are current and ex-smokers compared with severe childhood-onset asthma, and the adult-onset group has a higher pack-year history [89].

\section{Allergic asthma}

Total IgE concentrations in asthma are not altered by smoking status [19, 31], whereas specific $\operatorname{IgE}$ sensitisation to some common environmental allergens is reduced in current smokers and ex-smokers with severe asthma compared with never-smokers with severe asthma [31]. Smoking status of individuals with mild atopic asthma does not influence the magnitude of the allergen-induced early response [90, 91], whereas the magnitude of the late response is reported to be reduced [91] or similar [90] in current smokers compared with nonsmokers [91].

\section{Aspirin-exacerbated respiratory disease}

Active smoking in adulthood and exposure to second-hand smoke in childhood are associated with an increased risk of developing aspirin-exacerbated respiratory disease [92, 93].

\section{Obesity-related asthma}

The prevalence and intensity of current or former cigarette smoking in obese or nonobese individuals with severe asthma are similar $[94,95]$. Both smoking and obesity are independent risk factors for poor asthma control and increased exacerbations [96]. A survey of US adults reported that current smoking increased the risk of symptoms, emergency room visits and missing work days due to asthma associated with obesity or physical inactivity [97]. It is postulated that obesity and cigarette smoking modify the clinical expression of asthma in women by increasing systemic inflammation [98].

\section{Occupational asthma}

In a Canadian prospective observational study, the proportion of current and ex-smokers was similar between people with occupational asthma and with non-work-related asthma [99]. Cigarette smoking at the time of diagnosis does not adversely influence the outcome of occupational asthma [100].

\section{Chronic rhinosinusitis}

In the Swedish general population, cigarette smoking is associated with a high prevalence of chronic rhinitis in both men and women and a low prevalence of allergic rhinitis in men [101]. Increased severity of sinusitis on CT imaging is associated with higher blood eosinophil counts, serum IgE levels, exhaled 
nitric oxide and serum periostin concentrations in both adult nonsmokers $(<10$ pack-years $)$ and smokers with asthma ( $\geqslant 10$ pack-years), suggesting that CT imaging evidence of severe sinusitis was associated with T-helper cell type 2 (Th2)-high inflammation irrespective of smoking status [102].

\section{Psychological factors}

Active cigarette smokers with severe asthma had higher Hospital Anxiety and Depression Scale scores than never-smokers or ex-smokers with severe asthma, suggesting that these subjects were experiencing a greater degree of emotional distress [31]. In the general population, smoking cessation is associated with reduced depression, anxiety and stress, and improved positive mood and quality of life, compared with continuing to smoke [103].

\section{Inflammatory phenotypes}

Eosinophilic inflammation is a risk factor for exacerbations and predicts a beneficial response to corticosteroids. Th2-high inflammation is closely associated with eosinophilic inflammation and predicts a favourable therapeutic response to biological agents targeting the Th2 cytokine pathway. A noneosinophilic phenotype is associated with current smoking and is poorly responsive to current therapies including corticosteroids [104]. Cigarette smoking might induce a Th2-low inflammatory phenotype, although little is known about the prevalence or importance of Th2-low inflammation in current or ex-smoker with asthma or associated clinical asthma phenotypes.

\section{Summary}

Some clinical and inflammatory phenotypes of asthma are influenced by smoking status, and different phenotypes of chronic airway disease occur in current and ex-smokers without spirometric COPD or a history of asthma. Establishing the overlap between the phenotypes and endotypes of asthma and smoking-induced airway disease with normal lung function and their influence in directing therapy requires future investigation.

\section{Management}

The management of adverse health effects caused by exposure to tobacco smoke in people with asthma and in current and ex-smokers without spirometric evidence of COPD involves interventions ranging from public health measures to control exposure to tobacco smoke, personal advice on smoking cessation and the use of appropriate targeted therapies. Beyond advice on smoking cessation, there is limited information on drug treatment of smokers with asthma and symptomatic smokers with normal lung function $[1,2]$. The management of systemic smoking-induced comorbidities, such as cardiovascular disease, should be as guided by national and local recommendations.

\section{Smoking cessation}

Smoking cessation is an important goal in the management of all cigarette smokers, including those with asthma and symptomatic smokers with normal lung function. There is limited evidence of the effectiveness of smoking cessation on clinical outcomes in asthma, and most studies include small numbers of participants and are of short duration. In some studies, symptoms, lung function or corticosteroid sensitivity improve and sputum neutrophils decrease in those who quit smoking successfully [22, 105-108]. Former smokers with asthma have better symptom control than current smokers with asthma [20, 69]. Despite the known adverse effects of active smoking in asthma, these individuals are no more likely to receive physician counselling regarding smoking cessation nor smoking cessation pharmacotherapy compared with the general smoking population [109]. Smokers with asthma have poor smoking cessation rates [110], and slower rates of decline in nicotine withdrawal symptoms and craving during smoking quit attempts [111]. The effect of smoking cessation on clinical variables in symptomatic smokers recruited to the COPDGene or SPIROMICS cohorts is not reported, although smoking cessation was associated with older age of participants recruited to COPDGene [16]. In a population of current smokers with and without COPD, chronic respiratory symptoms, including bronchitis, were associated with the intension to stop smoking in the next 30 days, suggesting that the presence of respiratory symptoms could be used to aid smoking cessation advice in smokers with normal lung function [112].

\section{Current pharmacological treatments}

Evidence is limited on the effectiveness of therapeutic interventions directed at improving clinical outcomes due to their exclusion from most clinical trials. Studies in asthma usually exclude current smokers or ex-smokers with a smoking history of $>10$ pack-years and studies in COPD exclude symptomatic current smokers or ex-smokers without spirometric evidence of COPD or with a smoking history of $<10$ pack-years. Clinical trials in smokers with asthma may provide some limited guidance on drug treatment for current and ex-smokers with chronic airway disease without COPD or asthma. Despite the presence of reduced 
sensitivity to low-dose inhaled corticosteroids, higher doses improve lung function [57], although many patients remain symptomatic. Targeting the small airways with extra-fine-particle inhaled corticosteroids may potentially impact favourably on the safety and efficacy of inhaled corticosteroids through improved total lung deposition and improved asthma control at lower daily doses than large-particle inhaled corticosteroid. Observational studies suggest that extra-fine-particle hydrofluoroalkane beclometasone may achieve better control at lower prescribed doses than large-particle inhaled corticosteroids [113], but to date, randomised controlled trials have not clearly demonstrated that equipotent doses of small-particle inhaled corticosteroids are more effective than large-particle inhaled corticosteroids. The addition of a long-acting $\beta_{2}$-agonist to an inhaled corticosteroid improves clinical outcomes in smokers with asthma $[61,114,115]$. The long-acting muscarinic antagonist tiotropium was included in the 2015 Global Initiative for Asthma guideline as an alternative add-on therapy for people with a history of exacerbations [2]. The generalisability of the findings of clinical trials of tiotropium to smokers with asthma and smoking-related airway disease with normal lung function is uncertain, since current smokers and ex-smokers with $>10$ pack-years were excluded from clinical trials of tiotropium in asthma and in some studies, participants had to have chronic airflow obstruction (post-bronchodilator FEV $1 \%$ pred $\leqslant 80 \%$ and FEV1/FVC $\leqslant 0.7)$ [116]. There is some support for the effectiveness of tiotropium in "real-life" populations of asthma that include current and ex-smokers [81]. In an exploratory clinical trial in smokers with asthma, low-dose theophylline added to inhaled corticosteroid resulted in improvement in lung function, suggesting the restoration of corticosteroid sensitivity in those treated with the combination [117]. In one controlled trial, asthmatic patients with a smoking history of $\leqslant 11$ pack-years tended to show greater symptom control with inhaled fluticasone propionate over a 6-month period, whereas those with a smoking history of $>11$ pack-years tended to show greater benefit with the leukotriene receptor antagonist montelukast [118].

There are no clinical trials of drug treatments in symptomatic smokers with normal lung function to guide management. It remains to be established whether therapies shown to be effective in the treatment of smokers with asthma, such as long-acting $\beta_{2}$-agonist and inhaled corticosteroid combinations, will also benefit symptomatic smokers with normal lung function. Despite the uncertainty around drug treatment in this group, many symptomatic volunteers without COPD recruited to large COPD cohorts were taking respiratory medications. In SPIROMICS, $42 \%$ were taking bronchodilators and $23 \%$ were taking inhaled corticosteroids [17]; in COPDGene, 20\% used respiratory medications [16]. There is an unmet need for effective treatments for smokers without COPD as this group experience considerable disability from chronic respiratory symptoms and are at increased risk of exacerbations.

\section{Conclusions and future directions}

In conclusion, chronic respiratory symptoms occur in current and ex-smokers without spirometric evidence of COPD due to smoking-induced airway diseases including chronic bronchitis, emphysema, small airway disease or asthma, or a combination of these diseases. These individuals are more likely to experience wheezing, breathlessness, limitation in exercise, chronic bronchitis, poorer quality of life, exacerbations and smoking-related comorbidities than healthy never-smokers. Corticosteroid insensitivity and a greater decline in lung function are features of the smokers with asthma subgroup. Airway inflammatory and structural changes in smoking-induced airway disease without COPD overlap with those found in smokers with asthma. Cigarette smoking is associated with worse clinical outcomes in some phenotypes of asthma. The management of the adverse health effects caused by exposure to tobacco smoke involves public health measures to control exposure to tobacco smoke, personal advice on smoking cessation and the use of appropriate targeted therapies. Evidence is limited on the effectiveness of therapeutic interventions directed at improving clinical outcomes in these groups of patients due to their exclusion from most clinical trials.

There are many uncertainties about this chronic airway group, including their classification, mechanisms, natural history and management [119]. Future research needs to establish what proportions of symptomatic current and ex-smokers with normal spirometry have smoking-induced airway disease alone or also have asthma or a mixture of subtypes of smoking-induced airway disease (figure 1). In addition to the considerable overlap in clinical features that exists in current and ex-smokers with asthma and smoking-related airway disease without spirometric COPD, it will be important to understand the overlap in inflammatory mechanisms. Studies will require to recruit large numbers of current and ex-smokers from the general population and for these individuals to undergo detailed clinical, imaging and immunological phenotyping and endotyping, including the employment of system biological approaches. Different subtypes may have different mechanisms for exacerbations and other clinical outcomes and require individualised targeted therapies. The natural history of different subtypes of smoking-induced airway disease with normal lung function may differ. It is important to know whether subtypes are predictive of the development of COPD. Interestingly, a 5-year follow-up of the COPDGene cohort with normal lung 
function found that acute respiratory events did not increase the decline in lung function [42]. Current smokers with adult-onset asthma are at risk of developing COPD, whereas although current smokers with a history of childhood-onset asthma can develop COPD, this appears not to be due to the effects of cigarette smoking [51, 52], suggesting that different clinical phenotypes of asthma may differ in the effects of tobacco smoke on lung function. It remains to be established whether the identification of asymptomatic patients with smoking-induced airway diseases and normal lung function will lead to improved outcomes [120]. Research is required to determine the benefit of therapeutic interventions in this symptomatic population, particularly those at increased risk of exacerbations. Drugs that could be assessed for efficacy in symptomatic smokers with normal lung function include therapies currently used in the treatment of COPD, such as long-acting bronchodilators, azithromycin and roflumilast as well as other novel therapies under development [106]. In the future, the entry criteria for clinical trials should reflect "real-life" populations that including current and ex-smokers of differing ages and smoking intensity.

\section{References}

1 Global Initiative for Chronic Obstructive Lung Disease. Global strategy for the diagnosis, management, and prevention of chronic obstructive pulmonary disease. 2016. www.goldcopd.org Date last accessed: October 5, 2016.

2 Global Initiative for Asthma. Global strategy for asthma management and prevention. 2016. www.ginasthma.org Date last accessed: October 5, 2016.

3 Cazzola M, Rogliani P. Do we really need asthma-COPD overlap syndrome? J Allergy Clin Immunol 2016; 138: 977-983.

$4 \quad$ Postma DS, Rabe KF. The asthma-COPD overlap syndrome. N Engl J Med 2015; 373: 1241-1249.

5 Bateman ED, Reddel HK, van Zyl-Smit RN, et al. The asthma-COPD overlap syndrome: towards a revised taxonomy of chronic airways diseases? Lancet Respir Med 2015; 3: 719-728.

6 Christenson SA, Steiling K, van den Berge M, et al. Asthma-COPD overlap: clinical relevance of genomic signatures of type 2 inflammation in chronic obstructive pulmonary disease. Am J Respir Crit Care Med 2015; 191: 758-766.

7 Alshabanat A, Zafari Z, Albanyan O, et al. Asthma and COPD overlap syndrome (ACOS): a systematic review and meta analysis. PLoS One 2015; 10: e0136065.

8 Kumbhare S, Pleasants R, Ohar JA, et al. Characteristics and prevalence of asthma/chronic obstructive pulmonary disease overlap in the United States. Ann Am Thorac Soc 2016; 13: 803-810.

9 To T, Stanojevic S, Moores G, et al. Global asthma prevalence in adults: findings from the cross-sectional world health survey. BMC Public Health 2012; 12: 204.

10 Centers for disease Control and Prevention. Percentage of people with asthma who smoke. 2016. www.cdc.gov/ asthma/asthma_stats/people_who_smoke.htm Date last accessed: October 5, 2016.

11 Mannino DM, Gagnon RC, Petty TL, et al. Obstructive lung disease and low lung function in adults in the United States: data from the National Health and Nutrition Examination Survey, 1988-1994. Arch Intern Med 2000; 160: 1683-1689.

12 Halldin CN, Doney BC, Hnizdo E. Changes in prevalence of chronic obstructive pulmonary disease and asthma in the US population and associated risk factors. Chron Respir Dis 2015; 12: 47-60.

13 Soriano JB, Davis KJ, Coleman B, et al. The proportional Venn diagram of obstructive lung disease: two approximations from the United States and the United Kingdom. Chest 2003; 124: 474-481.

14 Tan WC, Bourbeau J, Hernandez P, et al. Exacerbation-like respiratory symptoms in individuals without chronic obstructive pulmonary disease: results from a population-based study. Thorax 2014; 69: 709-717.

15 Marsh SE, Travers J, Weatherall M, et al. Proportional classifications of COPD phenotypes. Thorax 2008; 63: 761-767.

16 Regan EA, Lynch DA, Curran-Everett D, et al. Clinical and radiologic disease in smokers with normal spirometry. JAMA Intern Med 2015; 175: 1539-1549.

17 Woodruff PG, Barr RG, Bleecker E, et al. Clinical significance of symptoms in smokers with preserved pulmonary function. $N$ Engl J Med 2016; 374: 1811-1821.

18 Martinez CH, Diaz AA, Meldrum C, et al. Age and small airway imaging abnormalities in subjects with and without airflow in SPIROMICS. Am J Respir Crit Care Med 2017; 195: 464-472.

19 Çolak Y, Afzal S, Nordestgaard BG, et al. Characteristics and prognosis of never-smokers and smokers with asthma in the Copenhagen General Population Study. A prospective cohort study. Am J Respir Crit Care Med 2015; 192: 172-181.

20 Clatworthy J, Price D, Ryan D, et al. The value of self-report assessment of adherence. rhinitis and smoking in relation to asthma control. Prim Care Respir J 2009; 18: 300-305.

21 Thomson NC, Chaudhuri R. Asthma in smokers: challenges and opportunities. Curr Opin Pulm Med 2009; 15: 39-45.

22 Polosa R, Thomson NC. Smoking and asthma: dangerous liaisons. Eur Respir J 2013; 41: 716-726.

23 Thomson NC, Chaudhuri R, Livingston E. Asthma and cigarette smoking. Eur Respir J 2004; 24: 822-833.

24 Tan WC, Hague CJ, Leipsic J, et al. Findings on thoracic computed tomography scans and respiratory outcomes in persons with and without chronic obstructive pulmonary disease: a population-based cohort study. PLoS One 2016; 11: e0166745.

25 Kim T-B, Jang A-S, Kwon H-S, et al. Identification of asthma clusters in two independent Korean adult asthma cohorts. Eur Respir J 2013; 41: 1308-1314.

26 Forey BA, Thornton AJ, Lee PN. Systematic review with meta-analysis of the epidemiological evidence relating smoking to COPD, chronic bronchitis and emphysema. BMC Pulm Med 2011; 11: 36-36.

27 Reports of the Surgeon General. The Health Consequences of Smoking: 50 Years of Progress. Atlanta, US Department of Health and Human Services, Centers for Disease Control and Prevention, Coordinating Center for Health Promotion, National Center for Chronic Disease Prevention and Health Promotion, Office on Smoking and Health, 2014. 
Coogan PF, Castro-Webb N, Yu J, et al. Active and passive smoking and the incidence of asthma in the Black Women's Health Study. Am J Respir Crit Care Med 2015; 191: 168-176.

Eisner MD, Anthonisen N, Coultas D, et al. An official American Thoracic Society public policy statement: novel risk factors and the global burden of chronic obstructive pulmonary disease. Am J Respir Crit Care Med 2010; 182: 693-718.

Elbehairy AF, Guenette JA, Faisal A, et al. Mechanisms of exertional dyspnoea in symptomatic smokers without COPD. Eur Respir J 2016; 48: 694-705.

Thomson NC, Chaudhuri R, Heaney LG, et al. Clinical outcomes and inflammatory biomarkers in current smokers and exsmokers with severe asthma. J Allergy Clin Immunol 2013; 131: 1008-1016.

Chaudhuri R, McSharry C, McCoard A, et al. Role of symptoms and lung function in determining asthma control in smokers with asthma. Allergy 2008; 63: 132-135.

Halbert RJ, Natoli JL, Gano A, et al. Global burden of COPD: systematic review and meta-analysis. Eur Respir J 2006; 28: 523-532.

Pelkonen M, Notkola IL, Nissinen A, et al. Thirty-year cumulative incidence of chronic bronchitis and COPD in relation to 30-year pulmonary function and 40-year mortality: a follow-up in middle-aged rural men. Chest 2006; 130: $1129-1137$.

Lange P, Parner J, Vestbo J, et al. A 15-year follow-up study of ventilatory function in adults with asthma. N Engl J Med 1998; 339: 1194-1200.

Vonk JM, Jongepier H, Panhuysen CI, et al. Risk factors associated with the presence of irreversible airflow limitation and reduced transfer coefficient in patients with asthma after 26 years of follow up. Thorax 2003; 58: 322-327.

Cerveri I, Cazzoletti L, Corsico AG, et al. The impact of cigarette smoking on asthma: a population-based international cohort study. Int Arch Allergy Immunol 2012; 158: 175-183.

Harmsen L, Thomsen SF, Ingebrigtsen T, et al. Chronic mucus hypersecretion: prevalence and risk factors in younger individuals. Int J Tuber Lung Dis 2010; 14: 1052-1058.

Thomson NC, Chaudhuri R, Messow CM, et al. Chronic cough and sputum production are associated with worse clinical outcomes in stable asthma. Respir Med 2013; 107: 1501-1508.

Martinez $\mathrm{CH}$, Kim V, Chen Y, et al. The clinical impact of non-obstructive chronic bronchitis in current and former smokers. Respir Med 2014; 108: 491-499.

Eisner MD, Iribarren C. The influence of cigarette smoking on adult asthma outcomes. Nicotine Tob Res 2007; 9 : 53-56.

Dransfield MT, Kunisaki KM, Strand MJ, et al. Acute exacerbations and lung function loss in smokers with and without COPD. Am J Respir Crit Care Med 2017; 195: 324-330.

Bowler RP, Kim V, Regan E, et al. Prediction of acute respiratory disease in current and former smokers with and without COPD. Chest 2014; 146: 941-950.

Murphy VE, Clifton VL, Gibson PG. The effect of cigarette smoking on asthma control during exacerbations in pregnant women. Thorax 2010; 65: 739-744.

Kauppi $\mathrm{P}$, Kupiainen $\mathrm{H}$, Lindqvist $\mathrm{A}$, et al. Long-term smoking increases the need for acute care among asthma patients: a case control study. BMC Pulm Med 2014; 14: 119-119. segmental airway lumen area in smokers with asthma. Chest 2015; 147: 735-744.

Bhatt SP, Soler X, Wang X, et al. Association between functional small airway disease and FEV1 decline in chronic obstructive pulmonary disease. Am J Respir Crit Care Med 2016; 194: 178-184.

Dilektasli AG, Porszasz J, Casaburi R, et al. A novel spirometric measure identifies mild COPD unidentified by standard criteria. Chest 2016; 150: 1080-1090.

Aanerud M, Carsin A-E, Sunyer J, et al. Interaction between asthma and smoking increases the risk of adult airway obstruction. Eur Respir J 2015; 45: 635-643.

Perret JL, Dharmage SC, Matheson MC, et al. The interplay between the effects of lifetime asthma, smoking, and atopy on fixed airflow obstruction in middle age. Am J Respir Crit Care Med 2013; 187: 42-48.

Hancox RJ, Gray AR, Poulton R, et al. The effect of cigarette smoking on lung function in young adults with asthma. Am J Respir Crit Care Med 2016; 94: 276-284.

Thomson NC. Does age of onset of asthma influence the effect of cigarette smoking on lung function? Am Respir Crit Care Med 2016; 194: 249-250.

Thomson NC. Addressing corticosteroid insensitivity in adults with asthma. Expert Rev Respir Med 2016; 10: 137-156. Barnes PJ. Corticosteroid resistance in patients with asthma and chronic obstructive pulmonary disease. J Allergy Clin Immunol 2013; 131: 636-645.

Heijink I, van Oosterhout A, Kliphuis $\mathrm{N}$, et al. Oxidant-induced corticosteroid unresponsiveness in human bronchial epithelial cells. Thorax 2014; 69: 5-13.

Chalmers GW, Macleod KJ, Little SA, et al. Influence of cigarette smoking on inhaled corticosteroid treatment in mild asthma. Thorax 2002; 57: 226-230.

Tomlinson JE, McMahon AD, Chaudhuri R, et al. Efficacy of low and high dose inhaled corticosteroid in smokers versus non-smokers with mild asthma. Thorax 2005; 60: 282-287.

Chaudhuri R, Livingston E, McMahon AD, et al. Cigarette smoking impairs the therapeutic response to oral corticosteroids in chronic asthma. Am J Respir Crit Care Med 2003; 168: 1308-1311.

Livingston E, Thomson NC, Chalmers GW. Impact of smoking on asthma therapy: a critical review of clinical evidence. Drugs 2005; 65: 1521-1536.

Telenga ED, Kerstjens $\mathrm{HA}$, ten Hacken $\mathrm{NH}$, et al. Inflammation and corticosteroid responsiveness in excurrent- and never-smoking asthmatics. BMC Pulm Med 2013; 13: 58.

Pedersen SE, Bateman ED, Bousquet J, et al. Determinants of response to fluticasone propionate and salmetero/ fluticasone propionate combination in the Gaining Optimal Asthma controL study. J Allergy Clin Immunol 2007; 120: $1036-1042$.

Dijkstra A, Vonk JM, Jongepier $\mathrm{H}$, et al. Lung function decline in asthma: association with inhaled corticosteroids, smoking and sex. Thorax 2006; 61: 105-110.

(ange P, Scharling $\mathrm{H}$, Ulrik CS, et al. Inhaled corticosteroids and decline of lung function in community residents with asthma. Thorax 2006; 61: 100-104. 
O'Byrne PM, Lamm CJ, Busse WW, et al. The effects of inhaled budesonide on lung function in smokers and nonsmokers with mild persistent asthma. Chest 2009; 136: 1514-1520.

65 Mannino DM, Davis KJ, DiSantostefano RL. Chronic respiratory disease, comorbid cardiovascular disease and mortality in a representative adult US cohort. Respirology 2013; 18: 1083-1088.

66 Oelsner EC, Hoffman EA, Folsom AR, et al. Association between emphysema-like lung on cardiac computed tomography and mortality in persons without airflow obstruction: a cohort study. Ann Intern Med 2014; 161: 863-873.

67 Ilmarinen P, Tuomisto LE, Niemelä O, et al. Comorbidities and elevated IL-6 associate with negative outcome in adult-onset asthma. Eur Respir J 2016; 48: 1052-1062.

68 Savage JH, Matsui EC, McCormack M, et al. The association between asthma and allergic disease and mortality: a 30-year follow-up study. J Allergy Clin Immunol 2014; 133: 1484-1487.

69 Boulet L, FitzGerald J, McIvor R, et al. Influence of current or former smoking on asthma management and control. Can Respir J 2008; 15: 275-279.

70 Westerhof GA, de Groot JC, Amelink M, et al. Predictors of frequent exacerbations in (ex)smoking and never smoking adults with severe asthma. Respir Med 2016; 118: 122-127.

71 Stampfli MR, Anderson GP. How cigarette smoke skews immune responses to promote infection, lung disease and cancer. Nat Rev Immunol 2009; 9: 377-384.

72 Kuschner WG, D’Alessandro A, Wong H, et al. Dose-dependent cigarette smoking-related inflammatory responses in healthy adults. Eur Respir J 1996; 9: 1989-1944.

73 Amin K, Ekberg-Jansson A, Löfdah CG, et al. Relationship between inflammatory cells and structural changes in the lungs of asymptomatic and never smokers: a biopsy study. Thorax 2003; 58: 135-142.

74 Soltani A, Ewe YP, Lim ZS, et al. Mast cells in COPD airways: relationship to bronchodilator responsiveness and angiogenesis. Eur Respir J 2012; 39: 1361-1367.

75 Berger KI, Pradhan DR, Goldring RM, et al. Distal airway dysfunction identifies pulmonary inflammation in asymptomatic smokers. ERJ Open Res 2016; 2: 00066-2016.

76 Niewoehner DE, Kleinerman J, Rice DB. Pathologic changes in the peripheral airways of young cigarette smokers. N Engl J Med 1974; 291: 755-758.

77 Demarche S, Schleich F, Henket M, et al. Detailed analysis of sputum and systemic inflammation in asthma phenotypes: are paucigranulocytic asthmatics really non-inflammatory? BMC Pulm Med 2016; 16: 1-13.

78 McSharry CP, McKay IC, Chaudhuri R, et al. Short and long-term effects of cigarette smoking independently influence exhaled nitric oxide concentration in asthma. J Allergy Clin Immunol 2005; 116: 88-93.

79 Broekema M, ten Hacken NH, Volbeda F, et al. Airway epithelial changes in smokers but not in ex-smokers with asthma. Am J Respir Crit Care Med 2009; 180: 1170-1178.

80 Ravensberg AJ, Slats AM, van Wetering S, et al. $\mathrm{CD}^{+} \mathrm{T}$ cells characterize early smoking-related airway pathology in patients with asthma. Respir Med 2013; 107: 959-966.

81 Price D, Kaplan A, Jones R, et al. Long-acting muscarinic antagonist use in adults with asthma: real-life prescribing and outcomes of add-on therapy with tiotropium bromide. J Asthma Allergy 2015; 8: 1-13.

82 St-Laurent J, Bergeron C, Pagé N, et al. Influence of smoking on airway inflammation and remodelling in asthma. Clin Exp Allergy 2008; 38: 1582-1589.

83 Wenzel SE. Asthma phenotypes: the evolution from clinical to molecular approaches. Nat Med 2012; 18 : 716-725.

84 Tuomisto LE, Ilmarinen P, Niemelä O, et al. A 12-year prognosis of adult-onset asthma: Seinäjoki Adult Asthma Study. Respir Med 2016; 117: 223-229.

85 Westerhof GA, Vollema EM, Weersink EJ, et al. Predictors for the development of progressive severity in new-onset adult asthma. J Allergy Clin Immunol 2014; 134: 1051-1056.

86 Tan DJ, Walters EH, Perret JL, et al. Clinical and functional differences between early-onset and late-onset adult asthma: a population-based Tasmanian Longitudinal Health Study. Thorax 2016; 71: 981-987.

87 Tan DJ, Walters EH, Perret JL, et al. Age-of-asthma onset as a determinant of different asthma phenotypes in adults: a systematic review and meta-analysis of the literature. Exp Rev Respir Med 2015; 9: 109-123.

88 Tommola M, Ilmarinen P, Tuomisto LE, et al. The effect of smoking on lung function: a clinical study of adult-onset asthma. Eur Respir J 2016; 48: 1298-1306.

89 Chaudhuri R, McSharry C, Heaney LG, et al. Effects of older age and age of asthma onset on clinical and inflammatory variables in severe refractory asthma. Respir Med 2016; 118: 46-52.

90 Meghji Z, Dua B, Watson RM, et al. Allergen inhalation challenge in smoking compared with non-smoking asthmatic subjects. Clin Exp Allergy 2011; 41: 1084-1090.

91 Cahn A, Boyce M, Mistry S, et al. Randomized trial of allergen-induced asthmatic response in smokers and non-smokers: effects of inhaled corticosteroids. Clin Exp Allergy 2015; 45: 1531-1541.

92 Chang JE, Ding D, Martin-Lazaro J, et al. Smoking, environmental tobacco smoke, and aspirin-exacerbated respiratory disease. Ann Allergy Asthma Immunol 2012; 108: 14-19.

93 Eriksson J, Ekerljung L, Bossios A, et al. Aspirin-intolerant asthma in the population: prevalence and important determinants. Clin Exp Allergy 2015; 45: 211-219.

94 Gibeon D, Batuwita K, Osmond M, et al. Obesity-associated severe asthma represents a distinct clinical phenotype: analysis of the British Thoracic Society difficult asthma registry patient cohort according to BMI. Chest 2013; 143: 406-414.

95 Holguin F, Bleecker ER, Busse WW, et al. Obesity and asthma: an association modified by age of asthma onset. J Allergy Clin Immunol 2011; 127: 1486-1493.

96 Yawn BP, Rank MA, Bertram SL, et al. Obesity, low levels of physical activity and smoking present opportunities for primary care asthma interventions: an analysis of baseline data from The Asthma Tools Study. NPJ Prim Care Respir Med 2015; 25: 15058.

97 Strine TW, Balluz LS, Ford ES. The associations between smoking, physical inactivity, obesity, and asthma severity in the general US population. J Asthma 2007; 44: 651-658.

98 Gibson PG. Obesity and asthma. Ann Am Thorac Soc 2013; 10: Suppl., S138-S142.

99 Lemière C, Boulet L-P, Chaboillez S, et al. Work-exacerbated asthma and occupational asthma: do they really differ? J Allergy Clin Immunol 2013; 131: 704-710.

100 Maestrelli P, Schlünssen V, Mason P, et al. Contribution of host factors and workplace exposure to the outcome of occupational asthma. Eur Respir Rev 2012; 21: 88-96. 
Eriksson J, Ekerljung L, Sundblad BM, et al. Cigarette smoking is associated with high prevalence of chronic rhinitis and low prevalence of allergic rhinitis in men. Allergy 2013; 68: 347-354.

Kimura H, Konno S, Nakamaru Y, et al. Sinus computed tomographic findings in adult smoking and non-smoking asthmatics: analysis of clinical indices and biomarkers. Ann Am Thorac Soc 2017; 14: 332-341.

103 Taylor G, McNeill A, Girling A, et al. Change in mental health after smoking cessation: systematic review and meta-analysis. BMJ 2014; 348: g1151.

104 Thomson NC. Novel approaches to the management of noneosinophilic asthma. Therap Adv Respir Dis 2016; 10: 211-234.

105 Chaudhuri R, Livingston E, McMahon AD, et al. Effects of smoking cessation on lung function and airway inflammation in smokers with asthma. Am J Respir Crit Care Med 2006; 174: 127-133.

106 Livingston E, Chaudhuri R, McMahon AD, et al. Systemic sensitivity to corticosteroids in smokers with asthma. Eur Respir J 2007; 29: 64-71.

107 Westergaard CG, Porsbjerg C, Backer V. The effect of smoking cessation on airway inflammation in young asthma patients. Clin Exp Allergy 2014; 44: 353-361.

108 McLeish AC, Zvolensky MJ. Asthma and cigarette smoking: a review of the empirical literature. J Asthma 2010; 47: 345-361.

109 Vozoris NT, Stanbrook MB. Smoking prevalence, behaviours, and cessation among individuals with COPD or asthma. Respir Med 2011; 105: 477-484.

110 Eisner MD, Yelin EH, Katz PP, et al. Predictors of cigarette smoking and smoking cessation among adults with asthma. Am J Public Health 2000; 90: 1307-1311.

111 McLeish AC, Farris SG, Johnson AL, et al. Evaluation of smokers with and without asthma in terms of smoking cessation outcome, nicotine withdrawal symptoms, and craving: findings from a self-guided quit attempt. Addict Behav 2016; 63: 149-154.

112 Melzer AC, Feemster LC, Crothers K, et al. Respiratory and bronchitic symptoms predict intention to quit smoking among current smokers with, and at risk for, chronic obstructive pulmonary disease. Ann Am Thorac Soc 2016; 13: 1490-1496.

113 Roche N, Postma DS, Colice G, et al. Differential effects of inhaled corticosteroids in smokers/ex-smokers and nonsmokers with asthma. Am J Respir Crit Care Med 2015; 191: 960-964.

114 Pilcher J, Patel M, Reddel HK, et al. Effect of smoking status on the efficacy of the SMART regimen in high risk asthma. Respirology 2016; 21: 858-866.

115 Clearie KL, McKinlay L, Williamson PA, et al. Fluticasone/salmeterol combination confers benefits in people with asthma who smoke. Chest 2012; 141: 330-338.

116 Kerstjens HA, Engel M, Dahl R, et al. Tiotropium in asthma poorly controlled with standard combination therapy. N Engl J Med 2012; 367: 1198-1207.

117 Spears M, Donnelly I, Jolly L, et al. Effect of low-dose theophylline plus beclometasone on lung function in smokers with asthma: a pilot study. Eur Respir J 2009; 33: 1010-1017.

118 Price D, Popov TA, Bjermer L, et al. Effect of montelukast for treatment of asthma in cigarette smokers. J Allergy Clin Immunol 2013; 131: 763-771.

119 Rodriguez-Roisin R, Han MK, Vestbo J, et al. Chronic respiratory symptoms with normal spirometry: a reliable clinical entity? Am J Respir Crit Care Med 2017; 195: 17-22.

120 Mannino DM. Does undiagnosed chronic obstructive pulmonary disease matter? Am J Respir Crit Care Med 2016; 194: 250-252.

121 Pinto LM, Gupta N, Tan W, et al. Derivation of normative data for the COPD assessment test (CAT). Respir Res 2014; 15: 68-68. 